\title{
Microanatomy of the Nerve to Iliacus Muscle and its Clinical Significance
}

\author{
Microanatomía del Nervio del Músculo Ilíaco y su Importancia Clínica
}

Rajani Singh'; Rajkumar²; Naresh Chandra³ ${ }^{3}$ Kavita Gupta ${ }^{4}$ \& M. S. Ansari ${ }^{1}$

SINGH, R.; RAJKUMAR; CHANDRA, N.; GUPTA, K. \& ANSARI, M. S. Microanatomy of the nerve to iliacus muscle and its clinical significance. Int. J Morphol., 37(3):1150-1163, 2019.

SUMMARY: The iliacus muscle, arising from iliac fossa is innervated chiefly by nerves to iliacus and femoral nerve. The tendon of iliacus muscle in the caudal part fuses with the tendon of psoas major muscle to form iliopsoas tendon As the iliacus/iliopsoas is responsible for flexing of the thigh and the forward tilting of the pelvis, body posture, Olympic lifts, daily activities like walking and running, so impairment of above functions, due to spinal cord injury or injury to nerves to iliacus, remained a grey area to explore manifestation of nerve lesions at fascicular level. Therefore an experimental study was designed to map the complex fascicular pathways suffering from splits, fusions and multiplexing coupled with measurement of distances of closely sampled histological slides. Tracking, correlation and interpretation of fascicles, in these slides of a cropped femoral nerve in iliacus region from a 70 year old female cadaver were analyzed. The study resulted in three schematic models of fascicular pathways in 3 nerves to iliacus and 2 tabular models of 2 remaining nerves to iliacus revealing complete picture of fascicles interrupted by dynamic transformational processes. These results would facilitate MRI neurographic interpretation at fascicular level and neurosurgical treatment through identification. The fascicular identification and setup would also discover anatomical complications and location of injury. Besides the huge data volume evolved off this experiment, the study would not only open up grey area for neuroanatomical research but also would revolutionize the neurosurgical repair and grafting of nerves to iliacus at fascicular level.

KEY WORDS: Fascicular pathways; Nerve lesion; Fascicle lesion; Iliacus muscle; Fascicular identification; Transformational processes.

\section{INTRODUCTION}

The flat and triangular iliacus muscle, an exact fit of the iliac fossa is located in the lower portion of the trunk. The tendon of iliacus muscle in the caudal part fuses with the tendon of psoas major muscle to form iliopsoas tendon. A two-third portion of this muscle is attached to the iliac fossa from its top. Another portion is attached to the inner portion of the iliac crest, the top, outer portion of the pelvic bone. Other fibers of this muscle are attached to the iliolumbar and anterior sacroiliac ligaments and up to the anterior iliac spines. These muscle fibers then converge and insert on the tendon at the lateral side of the psoas major muscle (Standring, 2008).

The iliacus is part of the hip rotator muscles which are responsible for the flexing of the thigh and the forward tilting of the pelvis. It is also one of the key muscles that help to maintain proper body posture. The iliopsoas contributes heavily to Olympic lifts and every day activities like walking and running. It also influences our form in many other types of movement, depending on how weak or how tight this group of muscles is. At the coxal joint, it flexes, laterally rotates, and adducts the hip.

The iliacus and iliopsoas muscles are innervated chiefly by branches of femoral nerve namely nerves to iliacus. These nerves to iliacus are made of motor and sensory fascicles/nerve fibers which communicate and control the activity or functions of iliacus and iliopsoas muscles. As these fascicles constituting nerves to iliacus are extending from iliacus and iliopsoas muscles to various control points in the brain through femoral nerve and spinal cord so in case of impairment of functions as illustrated above due to

\footnotetext{
${ }^{1}$ Department of anatomy, All India Institute of Medical Sciences Rishikesh Dehradun India.

${ }^{2}$ Department of Pediatric neurosurgery SGPGI Lucknow India.

${ }^{3}$ Department of Anatomy Hind Institute of Medical Sciences Lucknow India.

${ }^{4}$ Department of Physiology Santosh Medical College Ghaziabad India.
} 
spinal cord injury or injury to nerves to iliacus due to external or internal pathogens, toxins, drugs, environmental hazards, traumas, misuse of limbs, congenital anomalies and/or iatrogenic factors or otherwise, a challenge is thrown to the neurosurgeon for diagnosis and treatment of these insults because neither any information is available regarding the organization of these fascicular pathways in the nerve nor any internal morphological calibration of distance data from the known landmarks in this region.

The diagnostic process for malfunctioning of these muscles involves location and identification of injured nerve to iliacus and thereby corresponding damaged fascicles of the nerve. Though some anatomical and neurosurgical work on longitudinal organization and clustering of nerve fibers within fascicles of sciatic and peroneal nerves in the lower limb (Stewart, 2003) and pioneer work of Sundarland (1945) mainly on upper limb nerves have been carried out yet the longstanding controversy between somatotopy and plexiform structures of nerve fascicles (Stewart) remained a challenge to an anatomist and neurosurgeon. "This internal neural topography has substantial clinical relevance both in terms of clinical manifestations of nerve lesions and in the surgical repair of nerve injury" (Sunderland, 1945, 1978). The classic clinical approach to the localization of a peripheral nerve lesion is to determine the involved muscles and cutaneous areas. So the site of nerve lesion is analyzed based on anatomy of nerve branches. Clinical experience has shown that this simple approach based on the anatomy of the nerve branches can be extremely misleading as partial nerve damage can result in such restricted motor or sensory deficits that the lesion appears to be more distal than it actually is (Stewart). Such a lesion would be clinically indistinguishable from more distal lesion affecting just the motor branch to that muscle. Such mislocalization can lead to surgery in completely the wrong place. This problem may be solved by correlation and interpretation of configuration of fascicular organization longitudinally and in transverse sections" (Stewart).

The diagnosis and treatment of injury to the peripheral nervous system in general and nerve to iliacus in particular is one of the greatest challenge in orthopedic or neurosurgery because the injury may occur anywhere in complete pathways of neural elements right from entry point of nerve into iliacus muscle to the controlling nerve center in the brain. In the injured nerve, individual fascicular repair demands identification and isolation of fascicle as the fascicle is the smallest unit of nerve tissue that can be visualized and manipulated surgically. Individual fascicular grafting is uncommon but if clinically critical single fascicle can be identified and isolated, it may be taken up for repair. In group fasciculargrafting, every attempt is made to accurately deliver regenerating axons through the graft material to a matching fascicular group in the distal stump. Emphasis again is placed on appropriate fascicular matching (Payne, 2001).

This suggests that for diagnosis and neurosurgical repair of injury to fascicle belonging to nerves to iliacus essentially requires the identification, isolation, location, orientation, shape and size of injured fascicles. The position of nerve lesion at fascicular level is ascertained by MRI advanced neurography (Bäumer et al., 2012; Chhabra et al., 2013) and its interpretation coupled with signs and symptoms. But interpretation of MRI advanced neurography necessitate sound, detailed and precise anatomical knowledge of variant neural configuration of nerves to iliacus, their innervating pattern, fascicular structure and configuration of their pathways coupled with fascicular relationship with the nerve. This may be achieved by mapping of longitudinal and cross sectional correlation and interpretation of fascicular arrangement in femoral nerve continuing to nerves to iliacus through histological slides.

Therefore this study is aimed at revealing the macro/ micro fascicular anatomy of femoral nerve and its branches, nerves to iliacus by closely sampled histological slides in cranial and caudal directions. Though this is first and basic study on a single femoral nerve yet it will uncover a great deal of information regarding configuration of nerves/ fascicles. This will aid not only in diagnosing, repairing/ regenerating and grafting during neurosurgical interventions but also for improved MRI neurographic interpretation at fascicular level.

\section{Experimental procedures:}

Classification, innervation pattern and calibration: One right femoral nerve of a female cadaver aged 70 years, from the point of its exit from lateral margin of psoas major muscle to the last branching point of last nerve to iliacus was cropped and in addition to this the nerves to iliacus were also exposed from superficial to deep up to the point of entry into the iliacus muscle (Fig. 1). The gross in situ examination of this femoral nerve (Fig. 1) from this cadaver revealed that nerve branches, generally known as nerves to iliacus were five in number in this nerve network. These five nerves were defined and classified as first (uppermost) NI1, second NI2, third NI3, fourth NI4 and fifth NI5 (lowermost) nerve to iliacus in the iliacus region. These nerves emanated from lateral side of femoral nerve. As per the location of entry points of these nerves in the iliacus muscle, NI1, NI2, NI3 and femoral nerve itself might be innervating the upper and middle part of this muscle whereas NI4 and NI5 might be supplying lower part of iliacus/iliopsoas muscle. The detailed branching pattern was examined grossly by physical observation and microscopically through preparation and examination of 


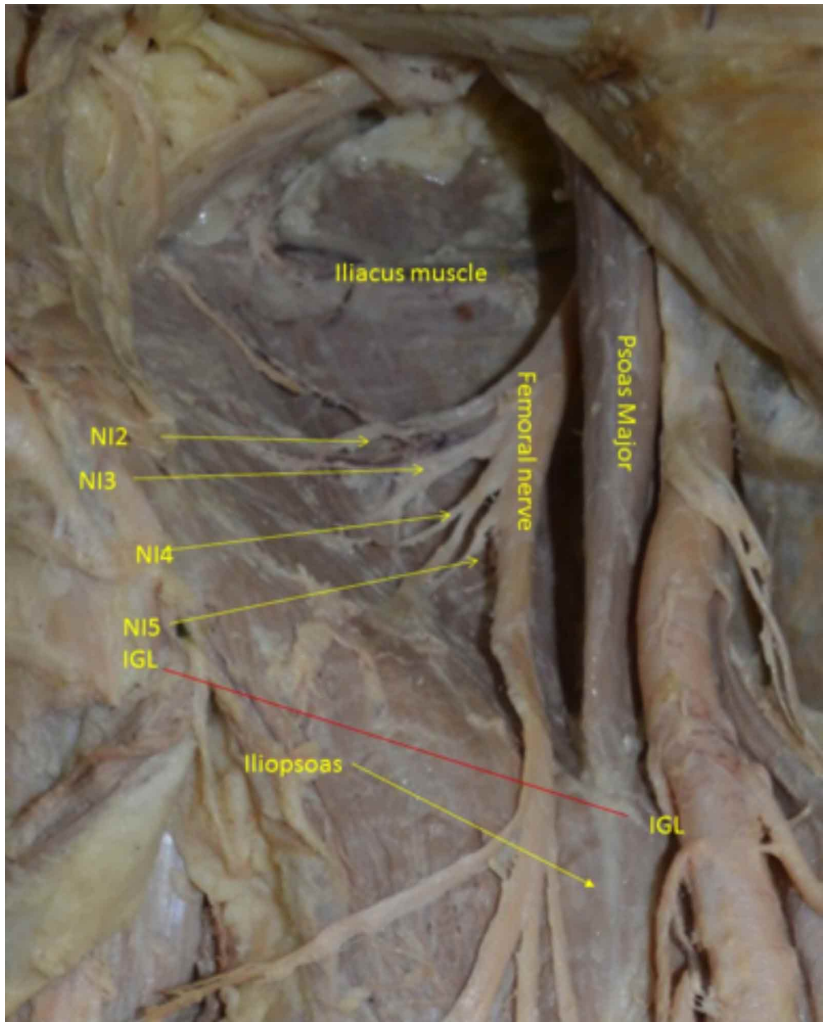

Fig. 1. showing in situ femoral nerve along with nerves to iliacus. I1, I2, I3, I4 and I5 are 1st, 2nd, 3rd, 4th and 5th nerves to iliacus. $\mathrm{IGL}=$ inguinal ligament.

histological slides. The physical measurement of points of interest such as top and bottom of the cropped femoral nerve segment and lengths of blocks coupled with point of emergence of nerves to iliacus was made with reference to inguinal ligament, a relevant landmark.

The slide plan: Thereafter the cropped femoral nerve was cut into 11 pieces (A1-A11) of which first two pieces (A1 and $\mathrm{A} 2$ ) of $5 \mathrm{~mm}$ length each and next nine (A3-A11) pieces of $4 \mathrm{~mm}$ length each were separated. These 11 pieces of femoral nerve were embedded in paraffin wax and 11 blocks were prepared for processing slides. In addition to this, the four blocks of $4 \mathrm{~mm}$ length each (B2, B3, B4 and B5) were also processed from NI2, NI3, NI4 and NI5.

Firstly, $1 \mathrm{~mm}$ length of the each block from the bottom was trimmed to make surface smooth. Then, first set of 2-7 slides of 5 micron thick sections from each block were cut from the bottom using microtome. Then, subsequently 2 to 4 sets of slides at the interval of $1 \mathrm{~mm}$ from each block were prepared. These slides were stained with hematoxylin and eosin. Then these sections were photographed by 18 megapixels Sony camera through faculty microscope. All the blocks were processed in similar manner. As reported by Sundarland (4), there are more frequent plexiform structures (in the interval of 2 to $3 \mathrm{~mm}$ ) in the proximal part of peripheral nerves so the plan of histological sectioning of femoral nerve has been so designed that maximum variations in fascicular configuration may microscopically be mapped keeping the sets of slides at the interval of $1 \mathrm{~mm}$. For precise/deterministic identification of nerve, fascicle, site and degree of injury and positions of these sections, the distances have been measured from inguinal ligament.

Slide Plan: Thus all the 11 blocks were processed from femoral nerve for 151 slides and 4 blocks (B2, B3, B4 and B5) from NI2, NI3, NI4 and NI5 for 59 slides to be correlated and interpreted by tracking fascicular pathways in femoral nerve and nerves to iliacus. Now the first slide from the top, A1 8 (here $\mathrm{A} 1$ is block number and 8 is slide's sequential number) was examined and all the composite fascicles were named from CFs 1 to 21 (Fig. 2). As long as the CF was consistent and continuous as correlated in different sections in cranial to caudal direction, the same number was continued but when any $\mathrm{CF}$ underwent transformational processes defined as splits, fusions and multiplexing, a new sequential identity number/s was/were given to new $\mathrm{CF} / \mathrm{s}$ so formed. For example- CF86 in section A9 1 split into new CFs 92 and 93 in Section A10 15 . Thus these two newly formed fascicles were numbered as above. The new number after fusion of CFs 59, 59' and 60 in A6 6 was given as CF46 in A6 7. CF38', CF63A, CF63B in A6 1 underwent multiplexing, changing shapes, sizes, location and orientation into new CFs, 38" and 63'A and 63'B in A7 11 due to intermingling and reorganizing of nerve fibers.

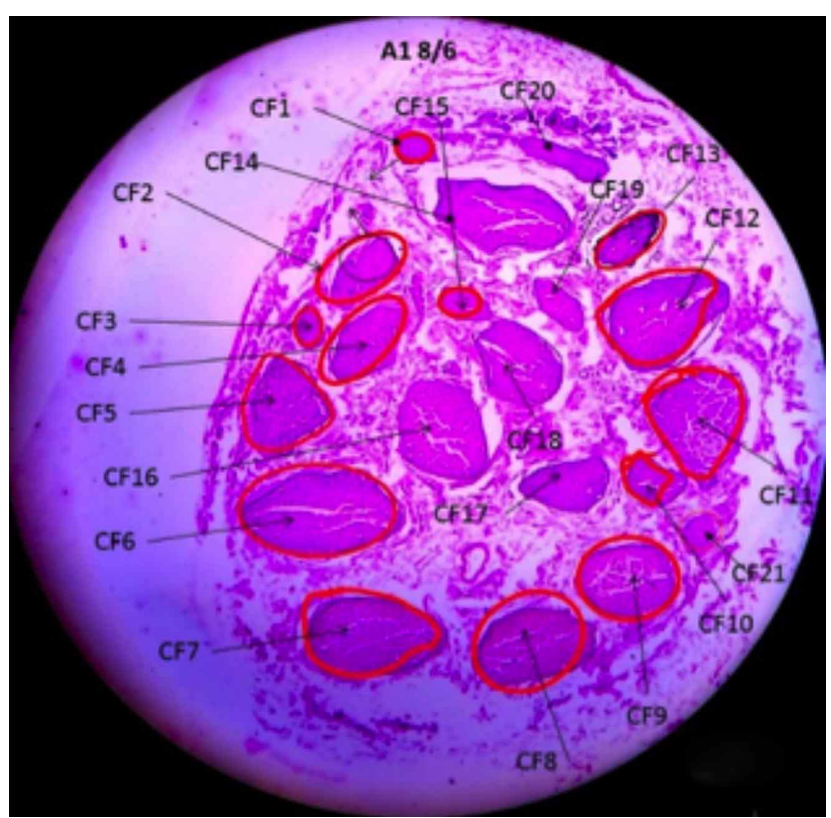

Fig. 2. showing 21 composite fascicles in upper most, $8^{\text {th }}$ slide of A1 block level (A1 8). The CFs were named from 1-21 e.g. CF1, CF2 etc. CF- composite fascicle. 
Correlation: Thus all the slides/sections were tracked, correlated and interpreted for identification, continuity and consistency interrupted by transformational processes of $\mathrm{CFs}$ from A1 8 through A11 1 in femoral nerve together with B2 15-1, B3 14-1, B4 15-1 and B5 15-1 in nerves to iliacus. The methodology adopted for correlation was placement of consecutive slides side by side caudally and cranially and comparing all the fascicles in both sections on the basis of correlation parameters. The parameters of correlation were, location (Dorsal, Ventral, Lateral and Medial; Central or peripheral), shapes (circular, oval and irregular), sizes (large, medium and small), orientation (Right handed, left handed, horizontal and vertical), split, fusion, migration and multiplexing (Intermingling of fibers of two or more CFs). As soon as intra-block (A1 8 to A1 1) correlation was over, the similar methodology was adopted for inter-block (A1 through A11) correlation through sequential slides. Thus all the sections were correlated and their pathways were identified from top slide to the emergence of nerve and then the correlation of composite fascicles in slides of branch nerves NI2, NI3, NI4 and NI5 innervating iliacus /iliopsoas muscles was also done in slides B2 15-1, B3 14-1, B4 15-1 and B5 15-1 in nerves to iliacus. This correlation was reconfirmed in the reverse direction from fascicles in the last slides (B2 1, B3 1, B4 1 and B5 1) of nerves to iliacus to the first slide (A1 8). The position of transformational processes including continuity and consistency were determined in the slides from its level to inguinal ligament as all the sections were calibrated in distance from inguinal ligament.

Modelling of fascicular pathways: Having mapped the CFs, manually hand drawn 3 schematic models of NI1, NI2 and NI3 were evolved showing the complete pathways of fascicles contributing to these nerves along with all the transformational processes in corresponding histological sections. This model was labelled at section levels with distances from inguinal ligament. For NI4 and NI5 nerves, the fascicular mapping was presented in form of tables (Table I and II, respectively) calling them 'Table Models' of NI4 (Table I) and NI5 (Table II) as due to presence of many most complex plexiform structures, the schematic diagram becomes very complicated and confusing as regards fascicular pathways.

\section{RESULTS}

Based on physical examination, there were five nerves to iliacus emanating from lateral side of femoral nerve. The uppermost, NI1 was emerging at the distance of 8.1, NI2 at 6.2 , NI3 at 5.2, NI4 at 4.8 and NI5 at $4.2 \mathrm{~cm}$, the lowermost one (Fig. 1). The tracking, correlation and interpretation of $\mathrm{CFs}$ revealed the formation and then the points of emanation of NI1 after A2 6 (Fig. 3), NI2 after A7 7 (Fig. 4), NI3 after A9 1 (Fig. 5), NI4 after A10 1 (Fig. 6) and NI5 after A11 1 (Fig. 7) were found at $8.10275,6.20475 \mathrm{~cm}, 5.20025 \mathrm{~cm}$, $4.80025 \mathrm{~cm}$, and $4.40025 \mathrm{~cm}$ respectively from inguinal ligament in femoral nerve based on calibration of histological sections. These distances established a good matching.

Observations and correlation of sections/slides. A total of 151 slides at a very close sample interval of 1 to $2 \mathrm{~mm}$ from 11 blocks of upper part of femoral nerve in the iliacus region were containing a total of more than 3000 fascicles undergoing
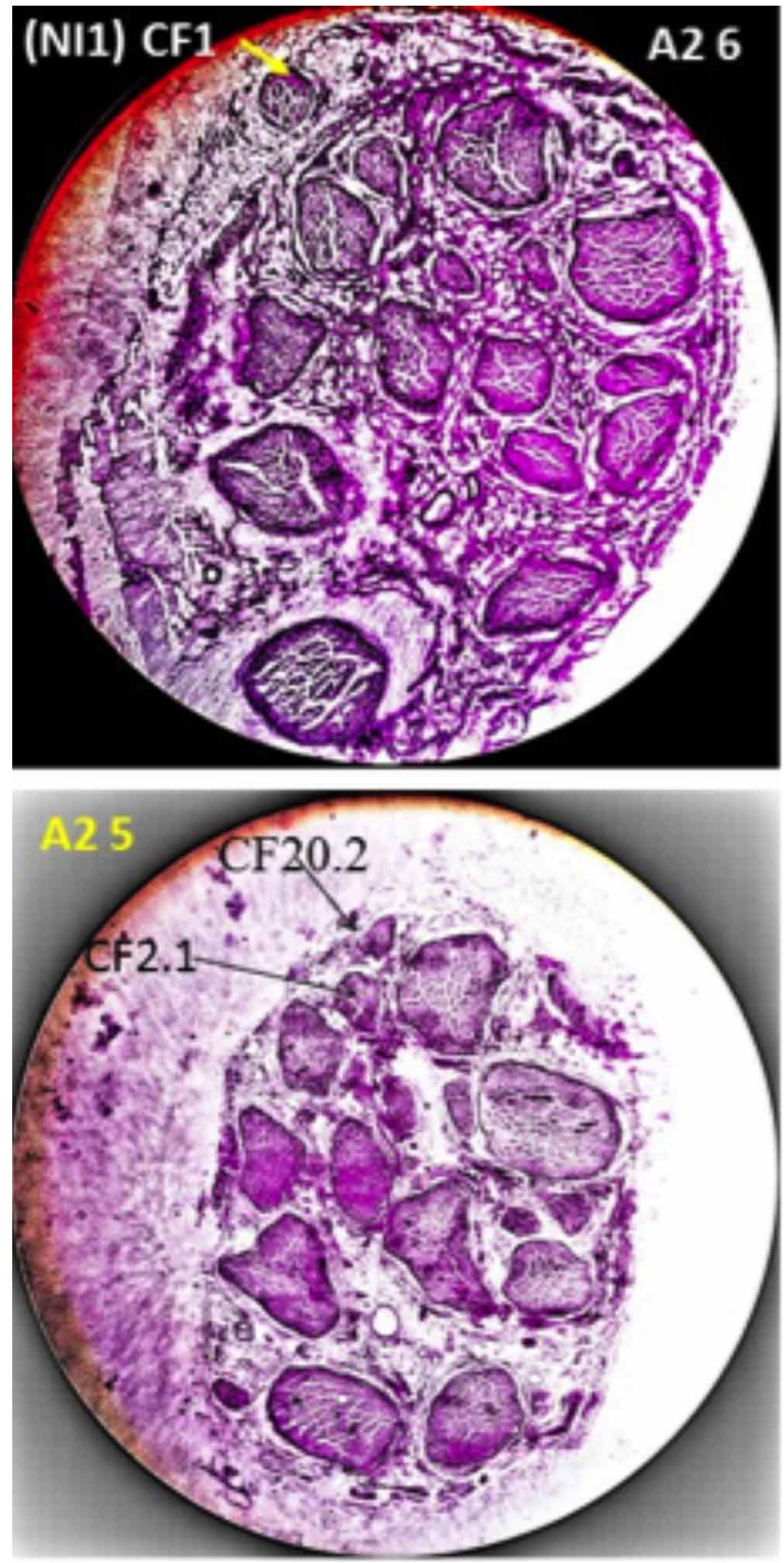

Fig. 3. showing emergence of I1 (first nerve to iliacus) consisting of CF1 (monofascicular nerve) seen in A2 6 and not present in A2 5 . 


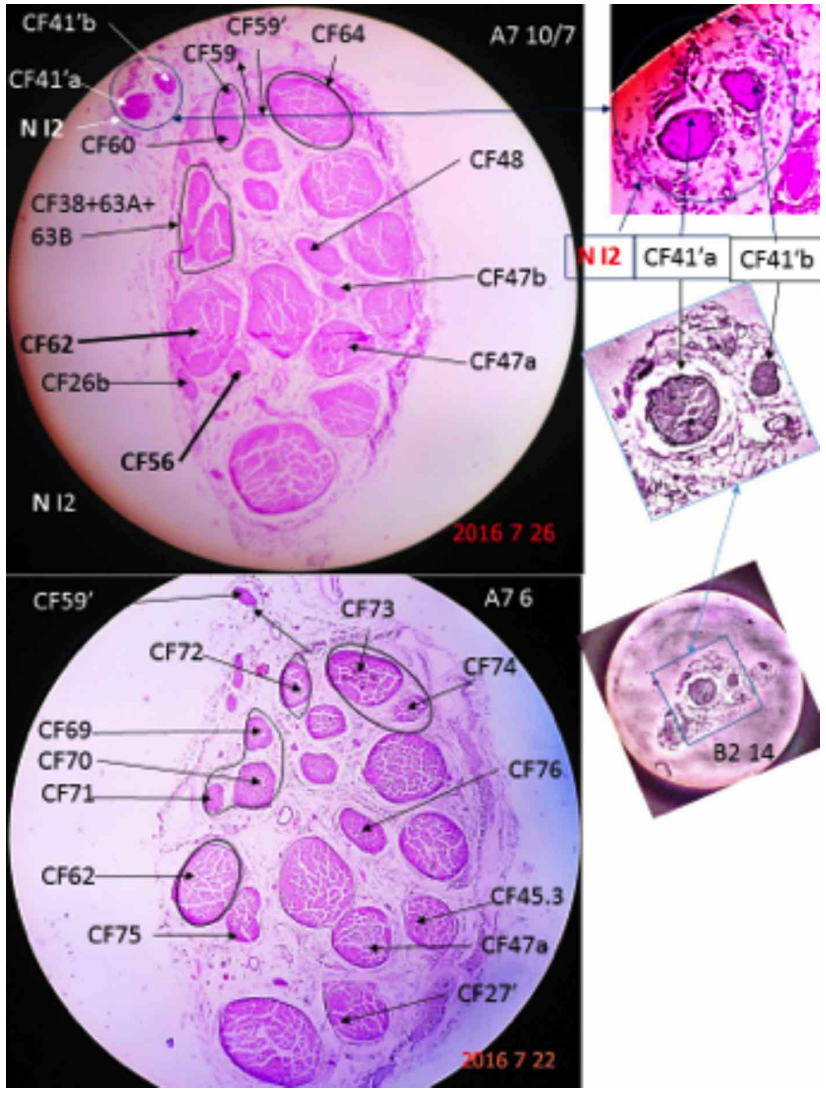

Fig. 4 showing emergence of I2, consisting of CF41'a and CF41'b, after slide A7 7 as these fascicles are surrounded by epineurium and are not seen in A7 6 of femoral nerve and the emergence is confirmed by correlation of CF41'a and CF41'b with CFs present in the slide B2 14 prepared from block B2 cut from 12 most proximally.

naturally occurring split, fusion and multiplexing processes. These processes took place for creating nerve branches to innervate organs. The presence of transformational processes in the fascicular pathways was causing changes in shape, sizes, location and orientation affecting the fascicular pathways. In addition to this, 59 slides from 4 blocks, B2, B3, B4 and B5 of NI2, NI3, NI4 and NI5 were prepared. The correlation of fascicles in these (B2 14, B3 15, B4 14 and B5 15) slides, closest to point of emanation of NI2, NI3, NI4and NI5 with the CFs forming these nerves in sections, (A7 7) (Fig. 4), (A9 1) (Fig. 5), (A10 1) (Fig. 6) and (A11 1) (Fig. 7) of femoral nerve confirmed the emergence of these nerves.

The correlation of CFs from the section A1 8 to A11 1 revealed that normally 2-7 sections of 5 microns thickness each, cut continuously in the same set were observed perfectly correlating with each other besides a few exceptions. The correlation was also found to exist between the slides of two consecutive sets except in case of multiplexing where other correlation parameters were used to establish the probable

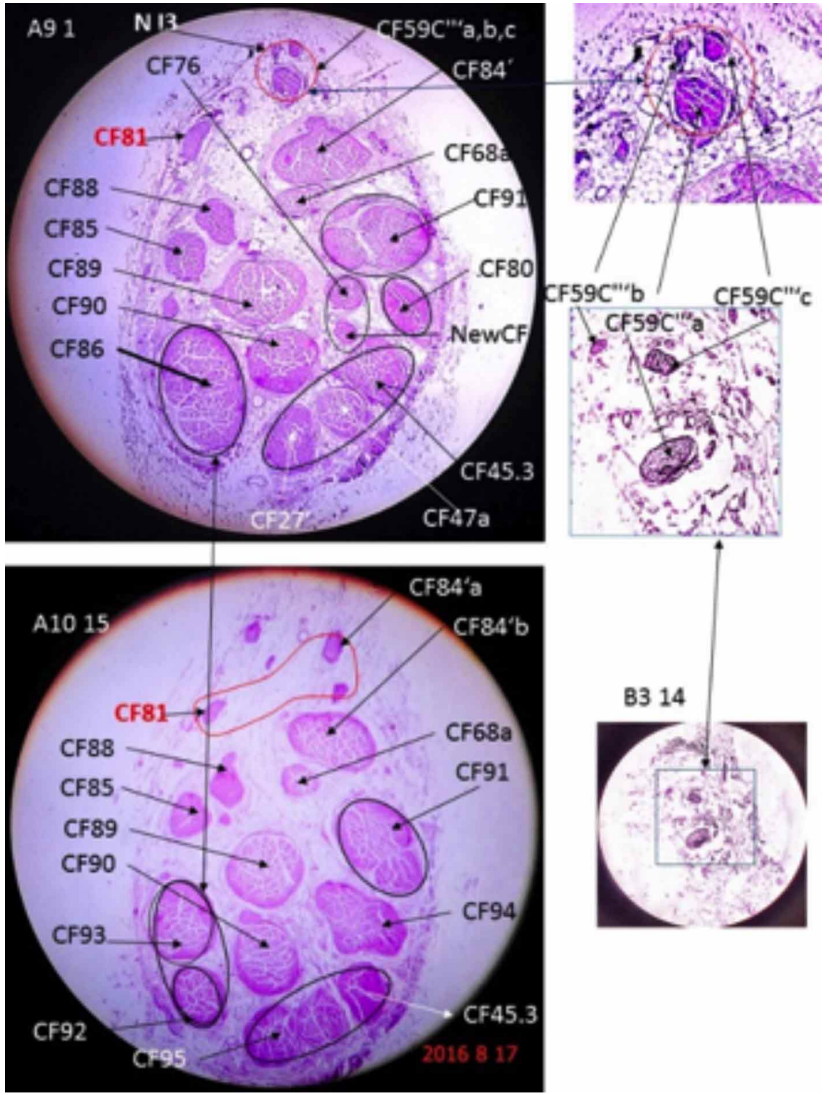

Fig. 5 showing emergence of I3 containing CFs 59" 'a, b, c after A9 1 as these fascicles are surrounded by epineurium and it is not visible in A10 15. The emergence is confirmed by slide B3 14 prepared from block B3 cut from I3 most proximally.

distribution of the nerve fibers. Normally the changes in these processes were regular but in a few cases, the transformational processes occurred instantly as observed in the consecutive slides at the interval of 5 micron. The consolidation of correlation, tracking and interpretation of the occurrences of these dynamic changes brought out 5 models (3 schematic and 2 tabular) of NI1, NI2, NI3, NI4 and NI5 nerves as illustrated below.

Model NI1 (Fig. 8): CF1 situated in the dorsal side in section A1 8 (Fig. 2) migrated laterally occupying dorsolateral position and then became consistent and continuous till section A2 6. The internal epineurium was seen surrounding $\mathrm{CF} 1$ and this $\mathrm{CF}$ was absent in section A2 5 (Figure 3). This established that NI1 was emanating out after section A2 6 . This was also confirmed by physical examination of emergence of NI1 with the help of calibrated distance. Thus CF1 constituting NI1 did not undergo any transformational processes. It clearly brings out the complete longitudinal pathway of CF1. 

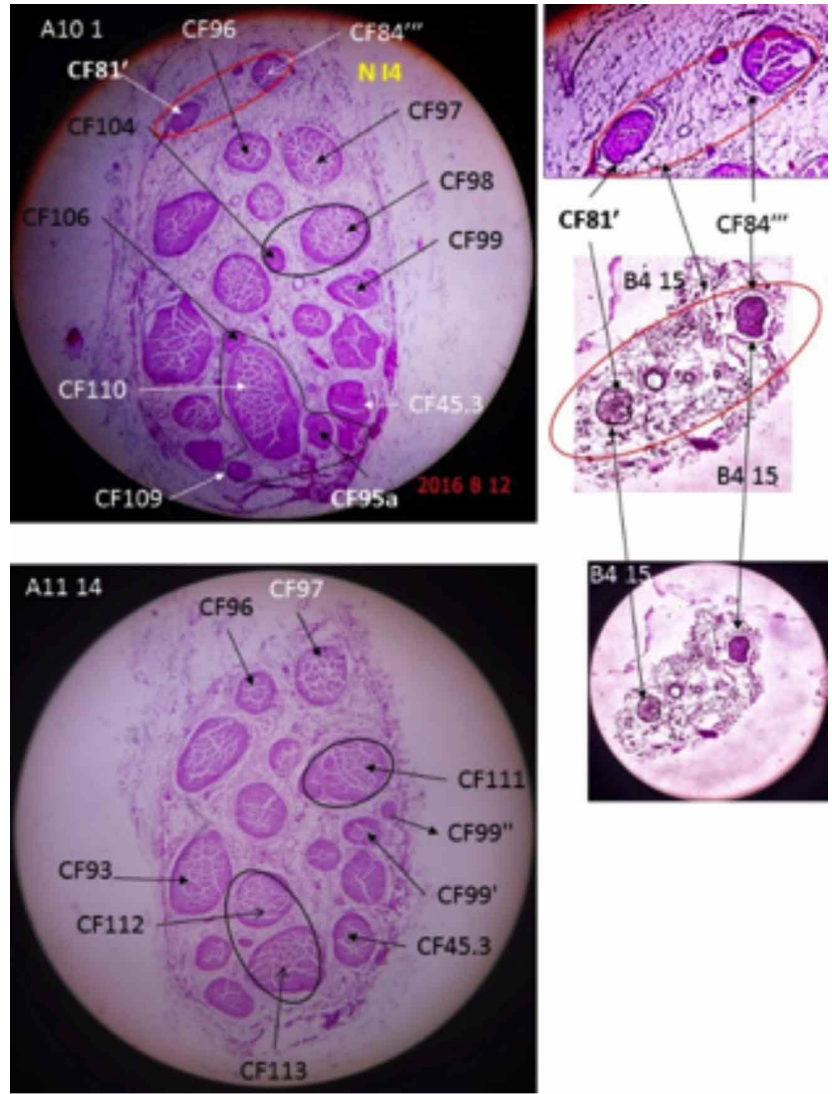

Fig. 6. Showing emergence of I4 consisting of CF84"' and CF81' after A10 1 as these fascicles are surrounded by epineurium and are not visible/absent in A11 14.The emergence is confirmed by slide B4 15 prepared from block B4 cut from I4.

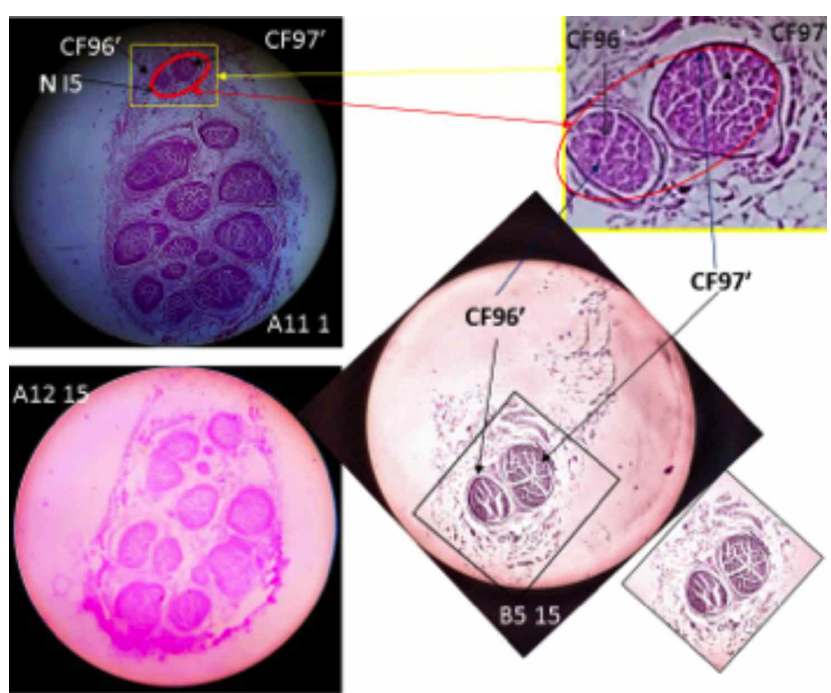

Fig. 7. Showing the nerve I5 constituted by CF96 and CF97 in A11 1 emerges out as it is not seen in A12 15 and is surrounded by epineurium. The emergence is confirmed by slide B5 15 prepared from block B5 cut from I5.

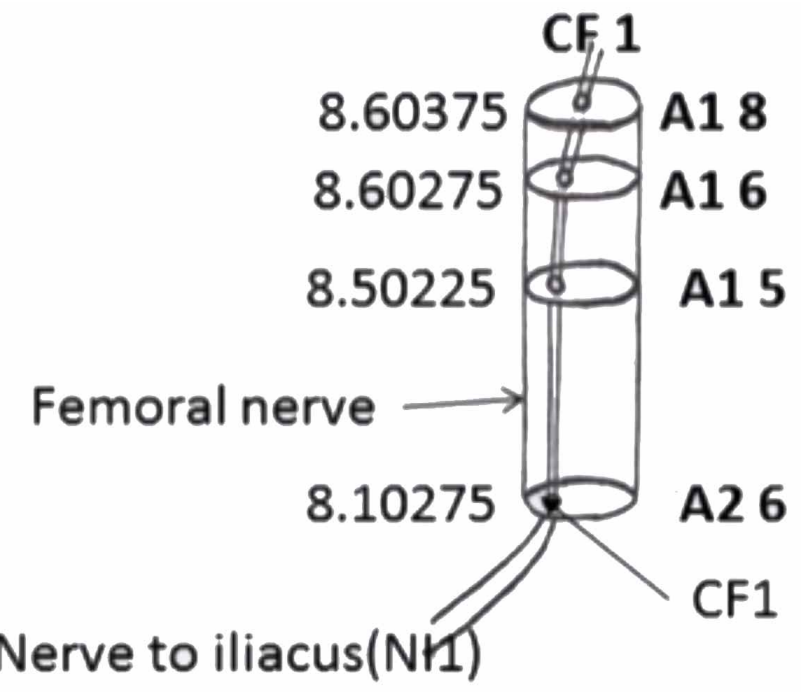

Fig. 8. Schematic diagram presenting formation and longitudinal pathway of I1 constituted by CF1 cranially from A1 8 to its emergence after A2 6. CF1=Composite Fascicle number 1 as defined in uppermost histological section A1 6/8 Fig. 1 and A1 8= 8th slide of block A1.

Model NI2 (Fig. 9): CF 20 in A1 6 split into CFs 20.1 and 20.2 in A1 5. The CFs 20.1 and 14 in A1 1 fused together forming CF25 in A2 19. CF25 in A3 1 split into CFs 25.1 and 25.2 in A4 14. CFs 20.2 and 25.1 in A4 11 fused together forming CF41 in A4 10. CF 41 in A4 6 split into CFs 41.1 and 41.2 in A4 5. CF41.2 in A4 1further split into CFs 41.2' and 41.2" in A5 12. CFs 41.2' and 41.2" in A6 7 fused together forming CF41' in A6 6. CF41' in A6 1 split into CFs 41' ${ }^{\prime}$ a and $41^{\prime} \mathrm{b}$ in A77. The internal epineurium was found surrounding CFs $41^{\prime} \mathrm{a}$ and $41^{\prime} \mathrm{b}$ in the process of formation of nerve NI2 and ready to emerge as these CFs were found absent in section A7 6. This revealed that it emergedout after the section A7 7 (Fig. 4). This was also confirmed by physical examination of emergence of nerve NI2 (Fig. 1). It was obvious from schematic diagram extracted from above histological slides that NI2 consisting of 41'a and 41'b in A7 7 evolved having undergone 5 splits and 3 fusions thereby redistributing/ regrouping and/or reorganizing the nerve fibers originally from CFs 14 and 20 in A1 8. The two fascicles after emergence were also observed in the closest slide B2 14 prepared from block B2 of NI2 (Figure 4) confirming the correlation of fascicular pathway.

Model NI3 (Fig. 10): As NI3 at point of emergence after A 91 contained CFs 59"' a, b and c surrounded by internal epineurium, consisting of nerve fibers contributed by original CFs 14, 15, 16 and 20 so the fascicular tracking and correlation envisaged that these CFs underwent 5 splits, 3 fusions and 2 multiplexing. These processes distributed/ regrouped and reorganized the nerve fibers into CF's $59^{\prime \prime \prime}$ a, 


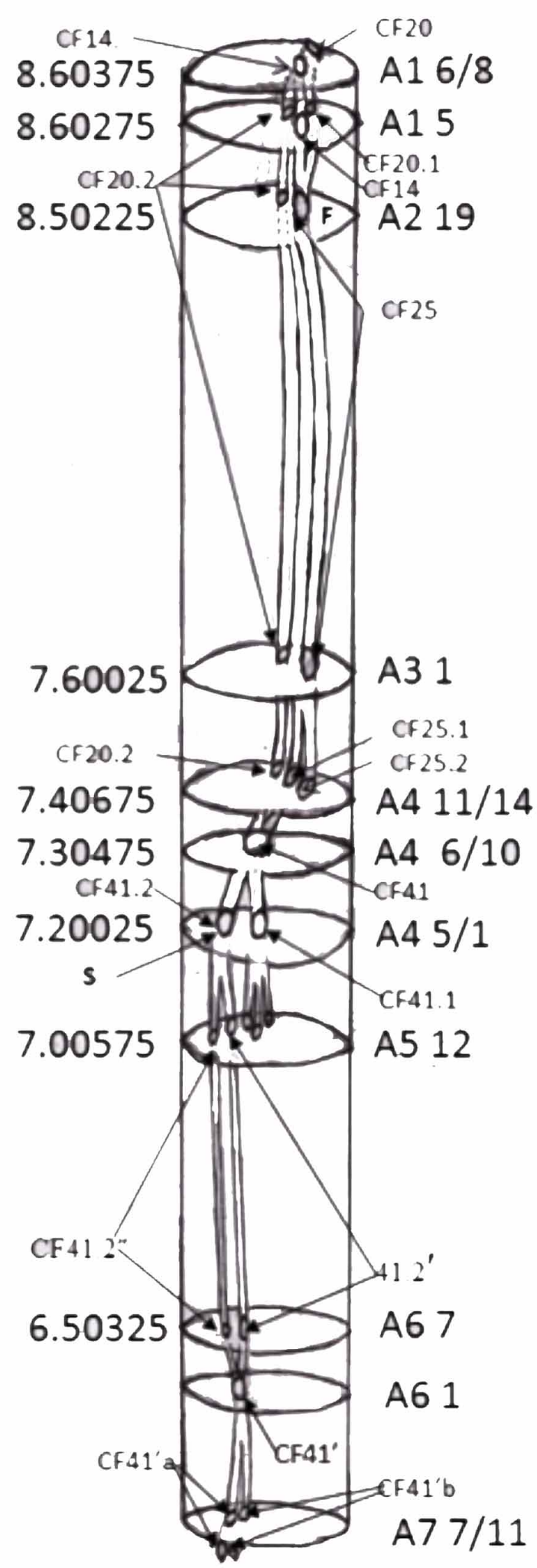

Nerve to iliacus (NI2)

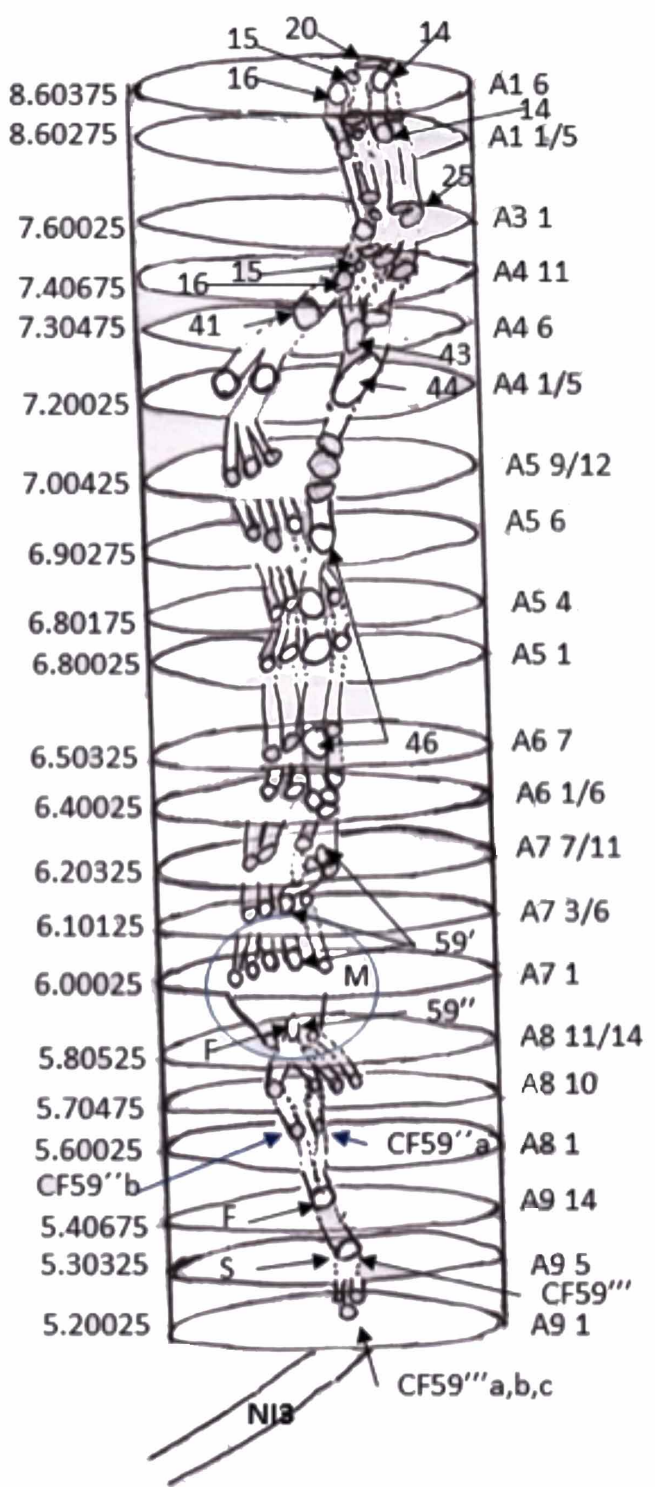

Fig. 10 Schematic diagram presenting formation and complete picture of longitudinal pathway of I3 (third nerve to iliacus) constituted by originally defined CFs $14,15,16$ and 20 cranially from A1 8 and undergoing splits, fusions and multiplexing ultimately transforming into CF59"'a, b, c constituting I3 at emergence after A9 1.

Fig. 9 Schematic diagram I2 (second nerve to iliacus) presents formation and complete picture of longitudinal pathways of CFs 41'a and 41'b Constituting I2 at emergence after A7 6. CFs 14 and 20 originally defined in A1 8, having undergone the transformational processes ( $S=5$ splits and $\mathrm{F}=3$ fusions), ultimately transforming into CFs $41^{\prime} \mathrm{a}$ and $41^{\prime} \mathrm{b}$, solely contribute to the fascicles of I2. The distances shown in the left hand side of nerve model are in centimetres and were measured from inguinal ligament. After undergoing splits and fusions marked on the diagram, the CFs were given a unique new name (e.g. 41'a) for identification as displayed in the diagram. $\mathrm{CF}=$ composite fascicle; $\mathrm{A} 18, \mathrm{~A} 76$---- are $8^{\text {th }}$ and $6^{\text {th }}$ slides of 1 and $7^{\text {th }}$ blocks respectively. 
$\mathrm{b}$ and $\mathrm{c}$ forming NI3. Thus, the tracking can be described as under-As CFs, 14 and 20 are common feeders of nerve fibers to both NI2 and NI3, so the contributions of CFs 14 and 20 and their derivatives are exactly same until the formation of CF 41 in A4 10 as illustrated in the model NI2. Remaining constituents CFs 15 and 16 are consistent till A4 11. Now CFs 15 and 16 in A4 11 fused together forming CF 43 in A4 10.CF 43 and 25.2 in A 46 fused to form CF 44 in A4 5. CF 44 in A4 1 split into CFs 44.1, 44.2, 44.3 in A5 12. CF 44.3 regrouped into CF 49 and CF 50 intermittently in A5 9. CF 44.2 and CF 49in A5 9 instantly transformed into CF 46 in A5 8. CF 46 in A6 7 split into CF 59, 59 $₫$ and 60 in A6 6. CF 41.1B $\phi$ in A7 3 split into 41.1B $\phi \phi \varnothing, 41.1 \mathrm{~B} \phi \phi$ in A7 2. CFs 59 $\phi, 41.1 \mathrm{~A} \phi$,
41.1B $\phi \varnothing \varnothing, 41.1 \mathrm{~B} \phi \varnothing$ and $41.1 \mathrm{C}$ in $\mathrm{A} 71$ underwent multiplexing transforming into CFs $59 \phi \varnothing, 41.1 \mathrm{~A}^{\prime \prime}$ and $41.1 \mathrm{C} \phi$ in $\mathrm{A} 814$. CF59 $\phi \varnothing$ in A8 11 split into CFs 59 $\phi \varnothing a$ and 59 $\phi \varnothing b$ in A8 10. CF 59 $\varnothing \varnothing a+C F 59 \phi \varnothing b$ in A8 1 fused together forming CF59 $\phi \varnothing \varnothing$ in A9 14. CF 59 $\notin \notin \varnothing$ in A9 5 split into CFs $59 \notin \notin \notin a, b$ and c in A9 4. NI3 constituted by CFs $59 \phi \varnothing \varnothing a, b$, c, emerged out after A9 1 as these fascicles were not observed in A10 15 (Fig.5). After emergence, NI3was also seen consisting of 3 fascicles, similar to CFs $59 \phi \phi \phi \mathrm{a}, 59 \phi \phi \phi b$ and $59 \phi \phi \phi c$ as observed in the closest slide B3 15 of block B3 of NI3 in Figure 5.

Table model NI4 (Table I): This model in form of table demonstrated the formation of nerve NI4 from contributions

Table I. Table NI4: Original CF's are the CFs named and defined in the uppermost slide of uppermost block A1: P is the processes; S= split, $\mathrm{F}=$ fusion and $\mathrm{M}=$ multiplexing: New CF's are the $\mathrm{CF}$ s given new unique sequential number/numbers after undergoing transformational process: Anr-Ams (Blocks), here An's and Am's are blocks represent the sequential block number and $\mathrm{r}$ and s stands for slide numbers, such as Anr is rth slide of Anth block: DAnr-DAms, DAnr and DAms represent distances of rth slide of Anth block and sth slide of Amth block from inguinal ligament.

\begin{tabular}{|c|c|c|c|c|}
\hline original (CF's) & $\mathrm{P}$ & New (CF’s) & Anr-Ams & DAnr-DAms \\
\hline $2,4,20,10,11,12,13,3,8,14,7,5,9,6,15,16$ & & Named and classified & A1 8 & 8.60375 \\
\hline $2,4,20: 10+11,12+13$ & $3 \mathrm{~S}: 2 \mathrm{~F}$ & $2.2+2.1,24+4^{\prime}, 20.1+20.2: 23,22$ & A1 6-A 15 & $8.60275-8.50225$ \\
\hline $2.2+3+4^{\prime}$ & $\mathrm{F}$ & 35 & A15-A 14 & $8.50225-8.502175$ \\
\hline $8: 14+20.1$ & $S: F$ & $8.1+8.2: 25$ & A1 1- A2 19 & $8.50025-8.30875$ \\
\hline $7+8.1$ & $\mathrm{~F}$ & 26 & A2 16-A2 15 & $8.30725-8.207$ \\
\hline $23,26: 5+24,8.2+9$ & $2 \mathrm{~S}: 2 \mathrm{~F}$ & $23.1+23.2,26 a+26 b: 36,27$ & A2 10-A2 9 & $8.2045-8.10425$ \\
\hline 23.1 & S & $29+30$ & A2 6-A2 5 & $8.10275-8.00225$ \\
\hline $6: 29+23.2$ & $\mathrm{~S}: \mathrm{F}$ & $32+33+34: 31$ & A2 1-A3 15 & $8.00025-7.80725$ \\
\hline $35: 36+32$ & $S: F$ & $35.1+35.2: 37$ & A3 6- A3 5 & $7.70275-7.60225$ \\
\hline $25,37: 35.1+2.1,22+30,26 a+34$ & $2 \mathrm{~S}: 3 \mathrm{~F}$ & $25.2+25 \cdot 1,37.1+37.2: 38,39,40$ & A3 1-A4 14 & $7.60025-7.40675$ \\
\hline $25.1+20.2,33+37.1, \mathbf{1 5}+\mathbf{1 6}$ & $3 \mathrm{~F}$ & $41,42,43$ & A4 11- A4 10 & 7.40525-7.30475 \\
\hline $41: 25.2+43,39+31,35.2+42$ & $S: 3 F$ & $41.1+41.2: 44,45,42$ & A4 6-A4 5 & $7.30275-7.20225$ \\
\hline $41.1,44,45: 40+27$ & 3S:M & $\begin{array}{l}41.1 \mathrm{~A}+41.1 \mathrm{~B}+41.1 \mathrm{C}, 44.1+44.2+44.3 \\
45.1+45.2+45.3: 40+27\end{array}$ & A4 1 A-5 12 & $7.20025-7.00575$ \\
\hline $44.2+44.3$ & M & $46+50$ & A5 9-A 58 & $7.00425-6.90375$ \\
\hline $38+41.1 \mathrm{~A}+41.1 \mathrm{~B}, 37.2+40^{\prime}$ & $2 \mathrm{M}$ & $38^{\prime}+41.1 \mathrm{~A}^{\prime}+41.1 \mathrm{~B}^{\prime}, 51+40^{\prime}$ & A5 5-A5 4 & $6.90225-6.90175$ \\
\hline $50+51$ & M & $54+55+56$ & A5 1-A6 15 & $6.80025-6.60725$ \\
\hline $42^{\prime}$ & S & $62+63$ & A6 12- A6 11 & $6.60575-6.50525$ \\
\hline 46,63 & $2 \mathrm{~S}$ & $60+59+59^{\prime}, 63 \mathrm{~A}+63 \mathrm{~B}$ & A6 7-A6 6 & $6.50325-6.40275$ \\
\hline $60: 38^{\prime}+63 \mathrm{~A}+63 \mathrm{~B}: 44.1+45.1$ & S:M:F & $67+68: 38^{\prime \prime}+63^{\prime} \mathrm{A}+63^{\prime} \mathrm{VB}: 64$ & A6 1-A7 11 & $6.40025-6.20525$ \\
\hline 59 & $\mathrm{CS}$ & 72 & A7 11 & 6.20525 \\
\hline $64: 26 \mathrm{~b}+56: 38^{\prime}+63^{\prime} \mathrm{A}+63^{\prime} \mathrm{B}$ & $S: F: M$ & $73+74: 75: 69+70+71$ & A7 7-A 76 & $6.20325-6.10275$ \\
\hline $41.1 B^{\prime}$ & $\mathrm{S}$ & $41.1 \mathrm{~B}^{\prime \prime}+41.1 \mathrm{~B}^{\prime \prime \prime}$ & A73-A7 2 & $6.10125-6.00075$ \\
\hline $67+72+73: 59^{\prime}+41.1 \mathrm{~A}^{\prime}+41.1 \mathrm{~B}^{\prime \prime}+41.1 \mathrm{~B}^{\prime \prime \prime}+41.1 \mathrm{C}$ & $\mathrm{F}: \mathrm{M}$ & $77: 59^{\prime \prime}+41.1 \mathrm{~A}^{\prime \prime}+41.1 \mathrm{C}^{\prime}$ & A7 1-A 814 & $6.00025-5.80675$ \\
\hline $62+75$ & M & $81+82+83$ & A8 11- A8 10 & $5.80525-5.70475$ \\
\hline $77+69$ & $\mathrm{~F}$ & 84 & A8 6- A8 5 & $5.70275-5.60225$ \\
\hline $84+41.1 \mathrm{~A}^{\prime \prime}+41.1 \mathrm{C}^{\prime}$ & $\mathrm{F}$ & $84^{\prime}$ & A $85-\mathrm{A} 84$ & $5.60225-5.60175$ \\
\hline $84^{\prime}, 81$ & $2 \mathrm{~S}$ & $84^{\prime} \mathrm{a}+84^{\prime} \mathrm{b}+84^{\prime} \mathrm{c}, 81^{\prime \prime}+81^{\prime \prime}$ & A9 1-A 1015 & $5.20025-5.00725$ \\
\hline $84^{\prime} \mathrm{a}+84^{\prime} \mathrm{c}$ & $\mathrm{F}$ & $84 "$ & A10 11-A 1010 & $5.00525-4.90475$ \\
\hline $84^{\prime \prime}+81^{\prime \prime}$ & $\mathrm{F}$ & $84 " '$ & A10 6-A10 5 & $4.90275-4.80225$ \\
\hline $84^{\prime \prime}+81^{\prime}$ & Emerged & AsNI4 after A10 1 & A 101 & 4.80025 \\
\hline
\end{tabular}


of CFs 2, 3, 4, 5, 6, 7, 8, 9, 10, 11,12, 13, 14, 15, 16, 20 and their derivative CFs (Fig. 2) through 23 splits, 25 fusions and 9 multiplexing events. As the CFs 14, 15, 16 and 20 are common feeders of nerve fibers to both NI3 and NI4 so the contributions of CFs 14, 15, 16, 20 and their derivatives are already illustrated in model NI3 and the same is applicable to NI4. The remaining CFs $2,3,4,5,6,7,8,9,10,11,12$ and 13 in A1 8 are consistent till A1 6. Then CFs 4 and 2 in A1 6 split respectively into CFs 4', 24 and CFs 2.1, 2.2 in A1 5. CFs 2.2, 3 and 4 ' in A1 5 fused together transforming into CF 35 in A1 4.CFs 5 and 24 in A2 10 fused together forming CF 36 in A2 9. CF 35 in A3 6 split into CF35.1 and 35.2 in $\mathrm{A} 35$. CFs 36 and 32 in A3 6 fused together forming CF37 in A3 5. CFs 25 and CF37 in A3 1 split into CFs 25.1 and 25.2; CFs 37.1 and 37.2 respectively in A4 14. CFs 37.1, 33 and CFs 25.1, 20.2 in A4 11 merged into CF42 and CF41 respectively in A4 10. CF41 in A4 6 split into CF41.1 and CF41.2 in A4 5 and CFs 35.2 and 42 in A4 6 merged into CF42' in A4 5. CF 41.1 in A4 1 split into CF41.1A, B,C in $\mathrm{A} 5$ 12.CF38, 41.1A and $\mathrm{B}$ in $\mathrm{A} 55$ underwent plexiformation transforming into $\mathrm{CFs} 38^{\prime}, 41.1 \mathrm{~A}^{\prime}$ and $41.1 \mathrm{~B}^{\prime}$ in A5 4. CF42' in A6 12 split into CF62, 63 in A6 11.

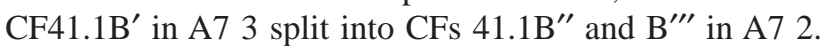

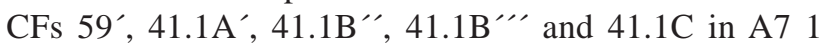
underwent multiplexing forming $\mathrm{CFs} 59^{\prime \prime}, 41.1 \mathrm{~A}^{\prime \prime}$ and 41.1C' in A8 14.CFs 62 and 75 in A811underwent plexiformation transforming into $\mathrm{CFs} 81,82$ and 83 in $\mathrm{A} 8$ 10. CFs $41.1 \mathrm{~A}^{\prime \prime}, 41.1 \mathrm{C}^{\prime}$ in $\mathrm{A} 85$ combined with 84 forming CF84' in A8 4. CF84' and CF81 in A9 1 split into CFs 84'a, $84^{\prime} \mathrm{b}$, and 84" and CFs 81' and 81' respectively in A10 15. CFs $84^{\prime} \mathrm{a}$ and $84^{\prime} \mathrm{c}$ in A10 11 merged together with 84' forming CF84" in A10 10. CFs 84" and 81" in A10 6 fused together forming CF84"' in A10 5. Now the nerve NI4 was formed of CFs $81^{\prime \prime}$ and $84^{\prime \prime \prime}$ as these CFs were observed surrounded by internal epineurium in A10 1 and were absent in the next section A11 14, establishing the emergence of NI4 after A10 1 (Fig. 6). This was further confirmed by correlation of CFs 81" and 84" with those observed in slide B4 15 of block B4 from proximal NI4.Physical examination of femoral nerve in relation to emergence of NI4 (Fig. 1) with the help of calibrated distance too confirmed this correlation.

Table model NI5 (Table II): The correlation, interpretation and tracking of fascicles, 2, 3, 4, 5, 6, 7, 8, 9, 10, 11, 12, 13, $14,15,16,17,18,20$ in A1 8and their derivatives in femoral nerve demonstrated that how these CFs contributed to NI5 consisting of CFs $96^{\prime}+97^{\prime}$ at the time of emergence after A 111 having undergone 28 splits, 29 fusions and 10 multiplexing events as illustrated in this table model NI5 (Table II). As the CFs 2, 3, 4, 5, 6, 7, 8, 9, 10, 11, 12, 13, 14, 15,16 , and 20 are common feeders of nerve fibers for both NI4 and NI5 and the pathways of CFs 2, 3, 4, 5, 6, 7, 8, 9,
$10,11,12,13,14,15,16$, and 20 were already illustrated in Table model.

The remaining CFs 17 and 18 in A1 8 were consistent till A2 6. CFs 17 and 18 in A2 6 fused together forming CF28 in A2 5. CF28 split into CFs 28.1 and 28.2 in A3 15. CF 28.2 in A5 6 split into CFs 47 and 48 in A5 5. CF 47 in A6 1 split into CFs 47a and 47b in A7 11. CFs 47a and 61 in A7 1 fused together forming CF 78 in A8 14. CFs 70 and 78 in A9 6 merged together forming $\mathrm{CF} 87$ in $\mathrm{A} 95$. CF 87 in A9 5 separated into CFs 88,89 in A9 4.CF 88 combined with CF 85 in A10 6 to form CF 103 in A10 5. CFs 96, 97 and 103 in A11 10 intermingle with each other forming CFs 96', 97', 97" and 114 in A11 9. Now CFs 96', 97' were found enveloped in internal epineurium and were not observed in A12 15. So these fascicles emerged out as NI5 after A11 1. These CFs 96', 97' forming nerve I5 in A11 1 were correlating with those of closest slide B5 15 of B5 of NI5 in Figure 7.

\section{Besides description of above results, some interesting observations were made as under.}

1.The transformational processes were frequent in the proximal region than in the distal part of femoral nerve.

2.The small sized CFs were seen more consistent and continuous than large sized ones.

3. The large sized CFs were found more prone to split than small sized ones.

4. There was clarity in distribution of nerve fibers in splits and fusion but the distribution in multiplexing was very complex.

\section{DISCUSSION}

The physical examination and measurements of cranial femoral nerve brought out NI1, NI2, NI3, NI4 and NI5 emerging out at 8.10275, 6.20475, 5.20025, 4.80025, and $4.40025 \mathrm{~cm}$ distances respectively from inguinal ligament from lateral side of femoral nerve. These nerves innervate iliacus muscle as detailed in previous section.

Microanatomy of nerves to iliacus. The mapping of longitudinal pathways of fascicles through tracking, correlation and interpretation of CFs in histological sections culminated into most comprehensible schematic and tabular models pertaining to NI1, NI2, NI3, NI4 and NI5, will not only facilitate to smoothen the controversy of somatotropy and plexiformation (Stewart) by identification, isolation and localization of injured fascicle and its relationship with the specific nerve but also provide shape, size and directivity of fascicle for matching of nerve fibers for repair, grafting 
Table II. Table NI5: Original CF's are the CFs named and defined in the uppermost slide of uppermost block A1 8: $\mathrm{P}$ is the processes; S= split, $\mathrm{F}=$ fusion and $\mathrm{M}=$ multiplexing: New CF's are the CFs given new unique sequential number/numbers after transformational process: Anr-Ams (Blocks), here An's and Am's are blocks represent the sequential block number and $\mathrm{r}$ and s stands for slide numbers, , such as Anr is rth slide of Anth block: DAnr-DAms, DAnr and DAms represent distances of rth slide of Anth block and sth slide of Amth block from inguinal ligament.

\begin{tabular}{|c|c|c|c|c|}
\hline Original (CF's) & $\mathrm{P}$ & New (CF's) & An r-Am s & DAn r-DAm s \\
\hline $\begin{array}{l}2,4,20,10,11,12,13,3,8,14,7,17,18,6 \\
15,16,9,5\end{array}$ & & Named and classified & A1 8 & 8.60375 \\
\hline $2,4,20: 10+11,12+13$ & $3 \mathrm{~S}: 2 \mathrm{~F}$ & $2.1+2.2,24+4^{\prime}, 20.1+20.2: 23,22$ & A $16-A 15$ & $8.60275-8.50225$ \\
\hline $2.2+3+4$ & $0 \mathrm{~S}: 1 \mathrm{~F}$ & 35 & A $15-\mathrm{A} 14$ & $8.50225-8.502175$ \\
\hline $8: 14+20.1$ & $1 \mathrm{~S}: 1 \mathrm{~F}$ & $8.1+8.2: 25$ & A1 1-A2 19 & $8.50025-8.30875$ \\
\hline $7+8.1$ & 0S: $1 \mathrm{~F}$ & 26 & A2 16- A2 15 & $8.30725-8.207$ \\
\hline $23,26: 8.2+9,5+24$ & $2 \mathrm{~S}: 2 \mathrm{~F}$ & $23.1+23.2,26 a+26 b: 27,36$ & A2 10-A2 9 & $8.2045-8.10425$ \\
\hline $23.1: 17+18$ & $1 \mathrm{~S}: 1 \mathrm{~F}$ & $29+30: 28$ & A2 6- A2 5 & $8.10275-8.00225$ \\
\hline $6,28: 23 \cdot 2+29$ & $2 \mathrm{~S}: 1 \mathrm{~F}$ & $33+32+34,28.1+28.2: 31$ & A2 1- A3 15 & $8.00025-7.80725$ \\
\hline $35: 32+36$ & $1 \mathrm{~S}: 1 \mathrm{~F}$ & $35.1+35.2: 37$ & A3 6-A 35 & $7.70275-7.60225$ \\
\hline $25,37: 22+30,35.1+2.1,26 a+34$ & $2 \mathrm{~S}: 3 \mathrm{~F}$ & $25.2+25.1,37.1+37.2: 39,38,40$ & A3 1- A4 14 & $7.60025-7.40675$ \\
\hline $15+16,25.1+20.2,37.1+33$ & 0S: $3 \mathrm{~F}$ & $43,41,42$ & A4 $11-$ A4 10 & $7.40525-7.30475$ \\
\hline $41: 39+31,25.2+43,42+35.2$ & $1 \mathrm{~S}: 3 \mathrm{~F}$ & $41.1+41.2: 45,44,42^{\prime}$ & A46- A4 5 & $7.30275-7.20225$ \\
\hline $41.1,44,45: 40+27$ & $3 \mathrm{~S}: 1 \mathrm{M}$ & $\begin{array}{l}41.1 \mathrm{~A}, \mathrm{~B}, \mathrm{C} ; 44.1,2,3 ; 45.1,2, \\
3: 40^{\prime}+27\end{array}$ & A4 1-A 512 & $7.20025-7.00575$ \\
\hline $44.2+44.3$ & $0 \mathrm{~S}: 1 \mathrm{M}$ & $46+50$ & A5 9-A 58 & $7.00425-6.90375$ \\
\hline $28.2: 38+41.1 \mathrm{~A}+41.1 \mathrm{~B}, 40^{\prime}+37.2$ & $1 \mathrm{~S}: 2 \mathrm{M}$ & $47+48: 38^{\prime}+41.1 \mathrm{~A}^{\prime}+41.1 \mathrm{~B}^{\prime}, 51+40_{-}^{\prime}$ & A5 6-A 55 & $6.90275-6.90225$ \\
\hline $40^{\prime}: 50+51$ & $1 \mathrm{~S}: 1 \mathrm{M}$ & $53+52: 54+55+56$ & A5 1-A6 15 & $6.80025-6.60725$ \\
\hline $42^{\prime-}$ & $1 \mathrm{~S}$ & $62+63$ & A6 12- A6 11 & $6.60575-6.50525$ \\
\hline $46,63: 53+54$ & $2 \mathrm{~S}: 1 \mathrm{~F}$ & $59+59+60,63 \mathrm{~A}+63 \mathrm{~B}: 61$ & A6 7- A6 6 & $6.50325-6.40275$ \\
\hline $60,47: 44.1+45.1: 38^{\prime}+63 A+63 B$ & $2 \mathrm{~S}: 1 \mathrm{~F}: 1 \mathrm{M}$ & $\begin{array}{l}\text { 67+68,47a+47b: } 64: 38 \_+ \\
\text {CF63_A+CF63_B }\end{array}$ & A6 1- A7 11 & $6.40025-6.20525$ \\
\hline 64: 38_'+CF63_A+CF63_B: $26 \mathrm{~b}+56$ & $1 \mathrm{~S}: 1 \mathrm{M}: 1 \mathrm{~F}$ & $73+7 \overline{4}: 69+70+\overline{7} 1: 75$ & A7 7- A7 6 & $6.20325-6.10275$ \\
\hline $41.1 \mathrm{~B}^{-}$ & $1 \mathrm{~S}: 0 \mathrm{~F}$ & $41.1 \mathrm{~B}_{-}^{\prime}+41.1 \mathrm{~B}_{-}^{\prime}$ & A7 3-A 72 & $6.10125-6.00075$ \\
\hline $\begin{array}{l}67+72 / 59+73,61+47 \mathrm{a}: \\
59+41.1 \mathrm{~A}^{\prime}+41.1 \mathrm{~B}^{\prime}+41.1 \mathrm{~B}^{\prime}{ }^{\prime}+41.1 \mathrm{C}\end{array}$ & $2 \mathrm{~F}: 1 \mathrm{M}$ & 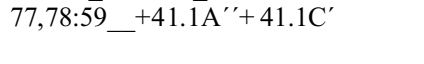 & A7 1-A8 14 & $6.00025-5.80675$ \\
\hline $62+75$ & 0S:1M & $81+82+83$ & A8 11-A8 10 & $5.80525-5.70475$ \\
\hline $77+69$ & $0 \mathrm{~S}: 1 \mathrm{~F}$ & 84 & A86-A8 5 & $5.70275-5.60225$ \\
\hline $71+82,84+41.1 \mathrm{~A}^{\prime \prime}+41.1 \mathrm{C}^{\prime}$ & $0 \mathrm{~S}: 2 \mathrm{~F}$ & $85,84^{\prime}$ & A8 5-A8 4 & $5.60225-5.60175$ \\
\hline $70+78$ & $0 \mathrm{~S}: 1 \mathrm{~F}$ & 87 & A96-A9 5 & $5.30275-5.30225$ \\
\hline 87 & $1 \mathrm{~S}: 0 \mathrm{~F}$ & $88+89+90$ & A9 5-A9 4 & $5.30225-5.20175$ \\
\hline $84^{\prime}$ & $1 \mathrm{~S}$ & $\mathrm{CF} 84^{\prime} \mathrm{a}+84 \_\mathrm{b}+84^{\prime} \mathrm{c}$ & A9 1- A10 15 & $5.20025-5.00725$ \\
\hline $84 \_b$ & $1 \mathrm{~S}: 0 \mathrm{~F}$ & $96+97$ & A10 11-A 1010 & $5.00525-4.90475$ \\
\hline $85+88$ & $0 \mathrm{~S}: 1 \mathrm{~F}$ & 103 & A10 6- A10 5 & $4.90275-4.80225$ \\
\hline $96+97+103$ & $0 \mathrm{~S}: 1 \mathrm{M}$ & $96+97+97^{\prime \prime}+114$ & A11 10-A11 9 & $4.60475-4.50425$ \\
\hline $96+97 \_$ & & NI $\overline{5}$ emerging after & A11 1 & 4.40025 \\
\hline
\end{tabular}

andregeneration (Payne). Besides this, our mapping brought out complete picture, labelled in distance from inguinal ligament, containing variant microanatomical elements such as consistency and continuity interrupted by splits, fusions and multiplexing, of CFs in their longitudinal pathways. The presence of these micro anatomical elements creates an element of indistinguishability in the longitudinal pathways of CFs so the knowledge of these variations is of paramount importance in identification of fascicles. Therefore, it is pertinent to elaborate neuroanatomical significance of transformational processes in terms of micro anatomical variations in the pathways of fascicles, distribution of nerve fibers and organization of CF's in formation of nerves to iliacus (NI1, NI2, NI3, NI4 and NI5) cranially from A1 8 to caudally up to point of emergence as a nerve branch of femoral nerve.

Split divide the CFs into two or more daughter CFs which contribute to one/two or more nerves innervating various structures adequately to suite the supply pattern by emerging and or evolving nerves. This process changes the shape/orientation and reduces the size of the fascicles due to distribution/redistribution of nerve fibers. The larger fascicles are more prone to splitting rather than small sized ones barring some exceptions.

Fusion causes mixing of two or more CFs resulting in one large $\mathrm{CF}$. This process introduces increase in shape, size coupled with change in location and orientation. So after fusion, if the CF contributes to one nerve before undergoing any transformational process, there is no regrouping of CFs further. As regards multiplexing, the nerve fibers of two or more CFs intermingle/regrouped in a very complex pattern transforming the shapes, sizes, locations and orientations of the CFs in such a way that the nerve fibers are divided, merged, redistributed, reorganized and regrouped in random pattern creating uncertainty in identification of fascicles therefore, it is very difficult if not impossible to identify the 
$\mathrm{CFs} /$ nerve fibers after this process. However, most probable major contribution of nerve fibers can always be ascertained. In such cases, the fascicles in question may be identified/located more precisely by intraoperative stimulation technique in association with the longitudinal and section wise fascicular interpretation of histological slides. Migration of fascicles causes the change in location leading to decussating of fascicles. This happens to affect adequate supply pattern by emanation of nerves. Besides occurrences of transformational processes, fascicles do show the consistency and continuity. This consistency and continuity is never along the whole pathway of fascicle/nerve fibers rather it is prevalent in between transformational processes. This establishes limited somatotopic structure of fascicles.

Single occurrence of transformational processes such as split, fusion and multiplexing as described above are simplistic processes but in actual practice, either the recurring occurrence of these single events or in combination with one another events in the same fascicles are prevalent. These incidences make the configuration/organization of the CFs more complex due to grouping/regrouping of $\mathrm{CFs}$ and distribution/redistribution of nerve fibers.

Analyzing the entire histological data of CFs consisting of above illustrated transient dynamic processes named as transformational processes prevalent in the longitudinal pathways of these CFs of all the five nerves to iliacus (NI1, NI2, NI3, NI4 and NI5), we evolved five models as illustrated below-Though these schematic models are not to the scale in relation to the size of CFs and localization of histological sections yet proportionate theme was kept intact in constructing the models. The table models presented the longitudinal pathways interrupted by split, fusion and multiplexing in each histological section shown in the table whereas in schematic models the complete longitudinal pictures of CFs was portrayed.

Schematic Model NI1: This model presents the sole and single composite fascicle (CF1), constituting NI1, is consistent and continuous without undergoing any of the transformational processes from A1 8 to the point of emergence of NI1 after section A2 6. Therefore there is no division and mixing of nerve fibers in CF1. Thus NI1 is a monofascicular nerve having somatotopic pattern of nerve fibers between sections A1 8 and A2 6. As illustrated above, this nerve innervate the upper part of iliacus muscle.

Schematic Model NI2: This model, extracted from correlated and interpreted histological sections of femoral nerve focusing on formation of NI2 consisting of two CFs $41 \phi \mathrm{a}$ and $41 \phi \mathrm{b}$ (situated dorsolateral at the brim of femoral nerve) at the instant of emergence of NI2, portrays complete picture of formation and emergence of NI2. The CFs $41^{\prime} \mathrm{a}$ and $41^{\prime} \mathrm{b}$ remain reliably continuous and consistent for $0.195 \mathrm{~cm}$. Then the CFs undergo two events of fusion and splitting without any change in total number of nerve fibers retaining their almost consistent position transforming into CF41.2. Up to this point not only fascicles but nerve fibers also may be considered identifiable and consistent. When CF41.2 merged with 41.1 forming CF41 in A4 6.Then the CF41 possesses additional fibers which are contributing to other nerves also. Here nerve fibers of NI2 intermixed together in CF41. The CFs, 41'a and 41'b constituting NI2 can also be correlated with the two fascicles present in slide B2 14 after the emergence of NI2. This confirms that NI2, after formation, emerged out after the section A7 11 in conformity with physical examination and calibration.

Schematic Model NI3: Our correlation and interpretation pertaining to the formation of nerve NI3, consisting of CF59"'a, b, c in this schematic model brings out the complete structure of fascicular pathways for their identification. If the formation of NI3 and its emanation is analyzed through interpreted pathways, the quantity of nerve fibers remains unchanged even after undergoing the events of splits and fusions up to the formation of CF59" in A8 14 for a distance of $0.6065 \mathrm{~cm}$ from point of emergence of NI3 cranially and are discernible and identifiable. The CF59"is the outcome of multiplexing in A8 14 receiving major contribution from CF59' and also from 41.1B" intermixing the nerve fibers. The CFs, 59"' a, b, c of NI3 were found correlatable reasonably well in B3 15 of NI3 even after its emergence. That confirms the identification and relation of fascicle with nerve.

Table model NI4 (Table I): This model illustrates the formation of NI4 with constituent CFs 81'and 84" longitudinally situated (dorsolateral) until its emergence after section A10 1 of femoral nerve. The entire data of tracking, correlation, interpretation for clarity in identification and comprehension of pathways of CFs are presented in Table model NI4 (Table I). This data brings out consistency and continuity interrupted by transformational processes very clearly wherever present in the model along with distances of all the processes through each calibrated histological section. It is pertinent to mention here that the smaller CF81ф is the result of splitting of CF81 which is continuous and consistent for fairly large distance between A8 10-A10 15 measuring to $0.7 \mathrm{~cm}$. The split of CF 81 in A10 15 distribute the nerve fibers in CFs 81' and 81". CF81" fuses with CF84" forming CF84"'. But CFs 84"' and 81' constitute the nerve NI4 so all the nerve fibers of CFs 81 and CF84" contribute solely to NI4. 
Table model NI5 (Table II): This model in form of table is presented to illustrate the formation of NI5 possessing CFs 96 4 and 97' (situated in dorsal side) and emerging after section A11 1 of femoral nerve. The CFs $96 \varnothing$ and 97' constituting NI5 are outcome of plexiformation of CFs 96,97 and 103. ThusNI5 received the major contributions from CFs 96, 97 with some intermingling of fascicles from 103.The complete picture of CFs, including all the dynamic and transient transformational processes in calibrated histological sides, has been presented in the table revealing all the micro anatomical changes in the configuration of fascicles. The CFs 96' and 97' are well correlatable with CFs present in the closest slide of B1 of NI5 establishing its emergence.

These models, depicting fascicular pathways through correlation, interpretation and longitudinal tracking in closely sampled histological sections, will enhance the degree of certainty in identification and location of the fascicles. If the analysis of information of micro anatomical variations in form of transformational processes in fascicular models of the longitudinal pathways of CFs calibrated in distances from inguinal ligament coupled with evolution of innervating nerves is integrated, this will revolutionize the crystal clear micro anatomical knowledge of pathways of fascicles.

Clinical significance: As illustrated in the introduction section, injury to nerve to iliacus thereby to the composite fascicles contained in the nerve may cause partial/full paralysis or weakness in the iliacus and or iliopsoas muscles impairing the functions of Olympic lifts, walking, running, flexing, hip rotation and other body postures. For such neural insults, the diagnosis of location and degree of injury pertaining to specific nerve/fascicles is imperative for planning surgical repair, grafting and regeneration. But the anatomists and neurosurgeons face the impediments in pinpointing the probable position of injury, the identification and localization of injured fascicle for intraoperative stimulation to confirm injured $\mathrm{CF}$ by muscle response, and its relationship with the specific nerve due to controversial somatotopy and or plexiform structures in the configuration, pattern and organization of CFs. The lack of thorough knowledge of innervation pattern illustrating the relationship of nerve to impaired muscle or problematic sensory area also hinders the diagnosis.

Therefore, before proceeding to detect the location of injury and affected elements (Nerve, fascicle and nerve fibers) for diagnosis, it is pertinent to know the category of injury whether external or internal. The external injury may damage the nerve and thereby fascicle or nerve fibers whereas, internally, injury may be created by compression and or degeneration by abnormal growth of harder tissues. Thus the injured elements may be nerve, fascicles and nerve fibers/ axons. But the fascicles in general and fascicles/nerve fibers of nerves to iliacus/femoral nerve in particular are the communicating channels of these insults to brain and its action plan in reverse direction to produce signs and symptoms. So it is not only nerves but also fascicles which can suffer from injury anywhere in entire fascicular pathways from origin to point of innervation producing similar signs and symptoms. Thus investigation should not be focused to nerves only rather longitudinal pathways and configuration of fascicles should also be kept in mind. Thorough understanding of clinical and functional Anatomy provides the clinician with the necessary tools for initial evaluation of the patient suffering from nerve injury (Payne). Therefore, in such cases, the neurosurgeon relates functional complication to neural/fascicular injury through analysis of innervation pattern of iliacus/iliopsoas muscles/part of these muscles by thorough neuroanatomical knowledge of network of nerves to iliacus keeping signs and symptoms in view to have a firsthand idea.

The detection of location and identification of nerve/ fascicular/nerve fibrous lesions and associated neural clinical complications are directly related to detailed neuroanatomy of nerve network in relation to its spatial nerve disposition and innervation pattern. This has been carried out under present study by detailed physical examination from gross and clinical anatomical view point, measurements and neuroanatomical description of nerves to iliacus network and its innervating pattern to iliacus muscle for establishing the relationship between problematic area/muscle dysfunction and the specific injured nerve.

Thus external injury is expressed but internal injury is occult. Therefore further diagnosis of expressed external injury requires knowledge of degree and location of injury which may be planned through focused MRI advanced neurography under the constraints of its limitation. But after preliminary investigation as elaborated above, the investigation of location of occult internal injury may be planned by ultrasound guided external nerve conduction study based on likely compressed or degenerated nerve due to presence of bony structures, vessels, hypertrophic muscles. This diagnosis can further be refined with laid down fascicular pathways portrayed in our NI1,NI2, NI3, NI4 and NI5 models. Further the location and degree of injury at fascicular level more accurately is carried out by MRI advanced neurography (Bäumer et al.; Chhabra et al.). This technique is most useful in locating the injury in fascicle but due to its limitations, it does not provide the correlation and identification of fascicle/nerve fibers in the innervating nerve. However, our models portraying the internal neural morphology of formation and emergence of NI1, NI2, NI3, NI4 and NI5 in and from femoral nerve may overcome this impediment as these models present the complete picture of 
CFs contributing to these nerves. The distance of location of injured fascicle from inguinal ligament may be computed in MRI neurography and then the calibrated histological section in the evolved models of NI1, NI2, NI3, NI4 and NI5 at the same level is compared and examined for confirmation of identification of injured fascicle. Besides, the analysis of the response of ailed organ by placing the electrodes at most suitable location for external/internal electrical stimulation of specific nerve/fascicle can be done. The success and non-invasion of stimulation will depend on finding out most suitable location for placement of electrical electrode. The most suitable site of placement of electrode may be decided on the basis of continuous and consistent fascicles contributing fully to the nerve innervating the problematic area from our models.

After identification of injured fascicle, the idea of shape, size, location and orientation can also be had from our model more so from histological section for matching, alignment and directivity of nerve fibers for repair and grafting besides analyzing from surgical procedure. As "The more precisely axons are directed toward their end organ, the better the chance for successful nerve regeneration. Intraoperative nerve stimulation in the awake patient is a readily available tool that can aid in this goal. Patient response to stimulation of selected fascicles in the proximal nerve stump can differentiate motor and sensory groups. Careful alignment is the critical factor in the first step. The nerve ends are inspected to identify fascicular groups matching proximally and distally amenable to individual fascicular/ nerve fiber repair" (Payne). These detailed procedures focus on longitudinal identification of fascicles, location of injury site and matching of fascicles for repair and grafting for administering the suitable surgical treatment. However, the neurosurgical manipulation is uncommon but it will be highly useful not only to diagnosis and neurosurgical intervention but also to develop fascicular electrode in case of fascicular repair.

Our models alert/caution to neurosurgeon to avoid intraoperative damage to fascicles/nerve fibers due to complex splits, fusions and multiplexing configuration of fascicles during neurosurgical manipulation for repair/ grafting to manage regeneration. Besides this, our models provide a leading role for precise interpretation of MRI neurography too. Planning of neurosurgical manipulations from MRI neurogrphy in association with our models will definitely enhance success to failure ratio drastically. As the presence of transformational processes is more frequent in the proximal part than in the distal one so the nerve lesion requires utmost attention in precise stimulation/repair or grafting in proximal part while the neurosurgeon will remain more comfortable in the distal part.

\section{CONCLUSION}

1.This is a very huge research work consisting of examination, analysis, tracking, correlation and interpretation of longitudinal pathways of more than 3000 fascicles in 200 histological slides (A1 8-A11 1 and B1 15 to B4 1) of femoral nerve and its branches, nerves to iliacus at a very close sample interval of 1-2 mm.

2. 5 fascicular models of five nerves to iliacus, NI1, NI2, NI3, NI4 and NI5 portraying complete picture consisting of detailed configuration, pattern, organization of fascicular pathways interrupted by splits, fusions and plexiforms, shapes and sizes (Fascicular anatomy) of CFs coupled with calibrated with distances from inguinal ligament.

3. The discovery of these occult micro anatomic variations in composite fascicular pathways of nerves to iliacus were revealed for the first time in the history of anatomic research. This would bring an unprecedented revolution in the field of neurosurgery of injured nerves to iliacus and femoral nerve.

4. The models open up new dimensions in precise interpretation of MRI advanced neurography.

5. Though this is a single study yet open up a large grey area for neuroanatomical and neurosurgical research. Therefore, these injuries with potentially devastating results provide ongoing stimulus for researchers striving for improved understanding of identification and location of injured fascicle in peripheral nerves in general and nerves to iliacus in particular.

6. Though fascicular surgery is presently, uncommon yet this study will be a milestone in tomorrow's rapidly evolving science and technology, specially, the fascicular electrode in line with nerve cuff electrode may be developed for minimal neurosurgical invasion.

\section{Recommendations}

1. More experiments are to be carried out on this portion of femoral nerves to know more variants of branching pattern of nerves to iliacus and corresponding fascicular configuration and their morphology in formation of these nerves.

2. The ability to manipulate nerve recovery at the cellular or gene level will provide the next significant improvement in nerve recovery. 


\section{Limitations}

1.The distribution of nerve fibers after plexiform structure is complex so identification of nerve fibers in resulting fascicle is subjective but interpretational skill and experience can minimize the ambiguity.

2. More experiments will produce more data for expanding scope of results of this study.

\section{ACKNOWLEDGEMENTS}

The authors are thankful to Mr Ram Manoj, technician for helping in the preparation of histological slides. There is no conflict of interest. This research did not receive any specific grant from funding agencies in the public, commercial, or not-for-profit sectors.

SINGH, R.; RAJKUMAR; CHANDRA, N.; GUPTA, K. \& ANSARI, M. S. Microanatomía del nervio del músculo ilíaco y su importancia clínica. Int. J. Morphol., 37(3):1150-1163, 2019.

RESUMEN: El músculo ilíaco, que se inserta en la fosa ilíaca, está inervado principalmente por los nervios ilíaco y femoral. El tendón del músculo ilíaco en la parte caudal se fusiona con el tendón del músculo psoas mayor para formar el tendón del músculo iliopsoas. Los músculos ilíaco e iliopsoas son responsables de la flexión del muslo y la inclinación hacia delante de la pelvis, la postura del cuerpo, los levantamientos olímpicos, las actividades diarias como caminar y correr, por lo que el deterioro de las funciones anteriores, debido a lesiones de la médula espinal o de los nervios ilíacos, constituyen una dificultad para explorar la manifestación de lesiones nerviosas a nivel fascicular. Por lo tanto, se diseñó un estudio experimental para mapear las complejas vías fasciculares que presentan divisiones, fusiones y multiplexación, junto con medición en muestras histológicas. Se analizó el seguimiento, correlación y la interpretación de los fascículos en muestras de secciones del nervio femoral en la región ilíaca de un cadáver femenino de 70 años. Se obtuvieron tres modelos esquemáticos de vías fasciculares en 3 ramos del nervio ilíaco y dos modelos tabulares de los 2 ramos nerviosos restantes del nervio ilíaco, que muestran una imagen completa de los fascículos interrumpidos por procesos de transformación dinámica. Estos resultados facilitarían la interpretación neurográfica de la resonancia nuclear magnética a nivel fascicular y el tratamiento neuroquirúrgico a través de su identificación. La identificación y configuración del fascículo también permitirían descubrir complicaciones anatómicas y la localización de la lesión. Además del enorme volumen de datos que se desprendió de este estudio, éste no solo contribuiría a la investigación neuroanatómica, sino también puede aportar a la reparación neuroquirúrgica y al injerto de nervios al músculo ilíaco a nivel fascicular.

PALABRAS CLAVE: Vías fasciculares; Lesión nerviosa; Lesión fascicular; Músculo ilíaco; Identificación fascicular; Procesos de transformación.

\section{REFERENCES}

Bäumer, P.; Heiland, S.; Bendszus, M. \& Pham, M. MR neurography Diagnostic criteria to determine lesions of peripheral nerves. Magnetom Flash, (Suppl. 2): 10-4, 2012.

Chhabra, A.; Zhao, L.; Carrino, J. A.; Trueblood, E.; Koceski, S.; Shteriev, F.; Lenkinski, L.; Sinclair, C. D. \& Andreisek, G. MR Neurography: Advances. Radiol. Res. Pract., 2013:809568, 2013.

Payne, S. H. Jr. Nerve repair and grafting in the upper extremity. J. South Orthop. Assoc., 10(3):173-89, 2001.

Standring, S. Gray's Anatomy. The Anatomical Basis of Clinical Practice. $40^{\text {th }}$ ed. Edinburgh, Churchill Livingstone/Elsevier, 2008. pp.1072.

Stewart, J. D. Peripheral nerve fascicles: anatomy and clinical relevance. Muscle Nerve, 28(5):525-41, 2003.

Sunderland, S. Nerves and Nerve Injuries. Edinburgh, Churchill Livingstone, 1978. pp.1046.

Sunderland, S. Traumatic injuries of peripheral nerves: I.-Simple compression injuries of the radial nerve. Brain, 68(1):56-72, 1945.

\author{
Corresponding author: \\ Dr Rajani Singh \\ Department of Anatomy \\ Additional Professor, AlIMS Rishikesh 249203 \\ Dehradun \\ INDIA
}

Email: nani_sahayal@rediffmail.com

Received: 10-06-2018

Accepted: 02-04-2019 